the membrane. B. Hille (University of Washington) summarised the evidence for such models for the sodium and potassium channels, as gleaned from the voltage clamp technique pioneered by Hodgkin and Huxley more than 20 years ago. Hille's and other data (Woodhull, J. gen. Physiol., 61, 687; 1973) suggest the presence of a carboxylic acid group inside the sodium pore.

The same carboxylic acid group was detected by J. M. Ritchie (Yale University) and colleagues, using a powerful technique not requiring electrical measurements ( $J$. Physiol., 232, 53P; 1973). They deduced its presence from the competitive binding of tritiated tetrodotoxin (TTX) and other ions to the sodium channel. TTX binding survives solubilisation of membranes in Triton X-100, but the sodium channels have yet to be purified by this route. By contrast, the much tighter binding of $\alpha$-bungarotoxin to acetylcholine receptors has aided their purification, as discussed by D. P. Green (University College, London).

The lack of a specific toxin for the potassium channel was widely lamented, though vagaries of the potassium currents in nodes of Ranvier and in cardiac muscle were described by $B$. Frankenhaeuser (Karolinska Institute, Stockholm) and D. Noble (University of Oxford). P. F. Baker (University of Cambridge) discussed delayed movements of calcium following action potentials, which he thinks are related to those activating transmitter release at synapses. L. G. M. Gordon and D. A. Haydon (University of Cambridge) talked about quantal permeability changes in artificial membranes, and G. K. Radda (University of Oxford) described the use of fluorescent probes.

A. von Muralt (Universität Bern) and J. V. Howarth (Marine Biological Association, Plymouth) summarised the slight optical and thermal changes in membranes during action potentials. These, it was concluded, reflect the consequences rather than the causes of the underlying permeability changes.

The latest and most controversial technique involves measuring the tiny displacement currents visible in voltageclamped axons when the usual ionic currents are blocked. C. M. Armstrong and F. Bezanilla (University of Rochester) think that these are 'gating currents', caused by the movement of those charged particules or dipoles thought to open and close the sodium channel (Nature, 242, 459; 1973), and they reported, as supporting evidence, that perfusion of an axon with $\mathrm{ZnCl}_{2}$ removed reversibly both sodium and displacement currents. Both currents could also be inactivated by depolarising pulses, and this effect was removed by pronase.

H. Meves (MBA, Plymouth) took issue with this interpretation, pointing out that other charged groups, which may vastly outnumber the grating groups, could easily obliterate the true gating current. Furthermore, although the qualitative behaviour of the measured displacement currents is acceptable, he observed that they depart from expectations in several quantitative respects. W. K. Chandler (Yale University), who first studied similar but slower currents in muscle (Nature, 242, 244; 1973), suggested that they play a part in excitationcontraction coupling.

If gating currents are what their name implies, then their magnitudes may be used to calculate the density of sodium channels, if one assumes six net charges per channel as given by Hodgkin and Huxley. R. D. Keynes (University of Cambridge), who organised the conference, and E. Rojas (MBA, Plymouth) estimated on the basis of both displacement currents (J. Physiol., 233, 28P; 1973), and TTX binding, that the squid giant axon has about 400 sodium channel per $\mu \mathrm{m}^{2}$. A. L. Hodgkin (University of Cambridge), using a dimensional argument, suggested that the density of sodium channels in nerve should be about 1,000 per $\mu \mathrm{m}^{2}$, if the conduction velocity is to be maximised. These numbers are much higher than the 13 channels per $\mu \mathrm{m}^{2}$ originally estimated on the basis of TTX binding to lobster nerve (Moore, Narahashi and Shaw, J. Physiol., 188, 99; 1967) and popularly subscribed to until about the middle of this meeting. The discrepancy suggests either vast species differences, or errors in the interpretation of TTX binding studies (tritiated impurities were mooted), or that 'gating' currents contain a large component that has nothing to do with gating. Caveat emptor.

\section{Cloning for obesity}

from our

Molecular Genetics Correspondent

NATURAL selection of organisms for traits desirable under particular circumstances is of course a well established feature of evolution. And artificial selection has been used both to isolate mutants of bacteria or eukaryotic cells in culture and to permit only cells possessing certain functions to survive. Cloning cells in culture can be used to isolate lines with enhanced or reduced expression of functions which can be followed quantitatively (rather than the all or nothing expression of many mutants). In a classic series of experiments some years ago, the 3T3 and 3T6 cell lines of mouse fibroblasts were derived with different responses to inhibition of growth by culture conditions. By cloning sublines of the 3T3 cell, Green and Kehinde now report in the March issue of Cell $(1,113 ; 1974)$ that they have isolated two sublines which accumulate large amounts of triglyceride fat in the resting state.

Illustrating their article with some striking colour photographs, Green and Kehinde demonstrate vividly that the cells of these sublines resemble adipose cells in accumulating large amounts of lipid in their cytoplasms. The initial observation which led to these experiments was the presence of a few cells possessing liquid droplets in resting cultures of $3 \mathrm{~T} 3$ cells; when resting cells are transferred to conditions which promote growth, the fatty cells are not found among the growing progeny, although such cells reappear when the culture again enters the resting state. By isolating areas rich in lipids from resting cultures, growing these isolates and then repeating the process when the culture reached the next resting phase, Green and Kehinde obtained cultures which were progressively richer in fatty cells. Eventually, they isolated two clones which accumulate lipid in large amounts.

When resting cultures of these cells are transferred to conditions which stimulate growth, the most fatty cells may survive for a few days but usually disintegrate eventually. The presence of large amounts of fat presumably interferes with the processes of cell division. But cells possessing less fat may reinitiate division; and after a successful division - sometimes mitosis fails in cytokinesis-the amount of lipid is diluted and then continues to decrease in each cell during successive cycles; except for the extremely fatty cells, therefore, both the lipid-accumulating sublines can be propagated indefinitely.

What is the cause of the lipid accumulation? Possible explanations, at present unresolved, include excessive uptake from the culture medium, increased synthesis within the cell or decreased degradation. Because the accumulation of lipid is inhibited by lipolytic agents such as dibutyryl cyclic AMP, Green and Kehinde suggest that one practical use of these lines may be to provide a means to test drugs for possible effects on lipid metabolism. And even more intriguing are some of the possible theoretical implications of the properties of these cells. What genetic change may underlie the conversion of a cell derived from the fibroblast into one apparently akin to the cells of adipose tissue? How is this change related to the usual processes of differentiation in developing mouse cells and can such changes be found for other phenotypes? The development of a differentiated phenotype in culture represents an important contrast with the more usual consequence of tissue culture. 